
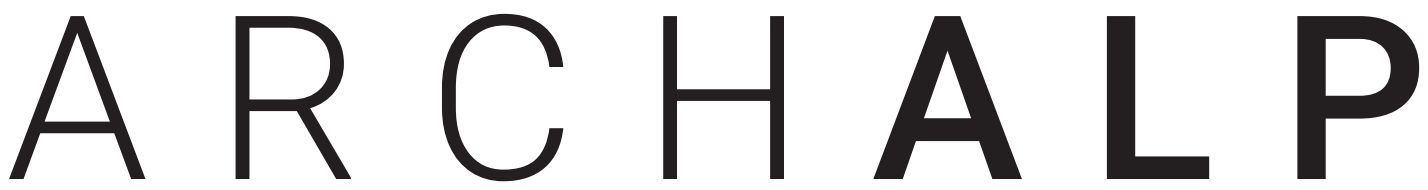

Rivista internazionale di architettura e paesaggio alpino / Revue internationale d'architecture et de paysage dans les Alpes / Internationale Zeitschrift für Alpine Architektur und Landschaft / Revija za alpsko arhitekturo in pokrajino / International journal of alpine architecture and landscape

Rer una nuota abitabilita delle Alpi.

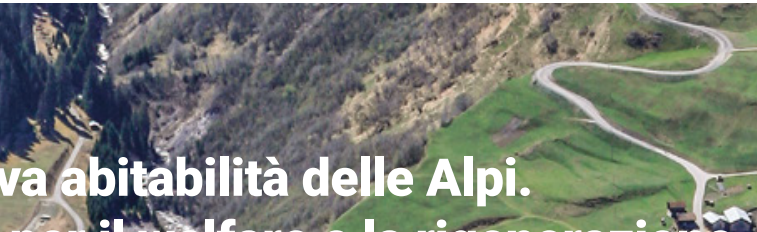
Architeture pet ilf lfare e la rigenerazione For a new inhabifability of the Alps. Architectures for welfare ând regeneration $/$ Pour une nouvelle habitabilité des Alpes. Architectures pour le welfare et la régéhérationy Für eine núue Bewohnbarkeit in den Alpen. Architekturen für Wohf ahrt und Regeneration / Za novo. bivalnost v Alpah, arhitekture za dobrobit in regeneracijo
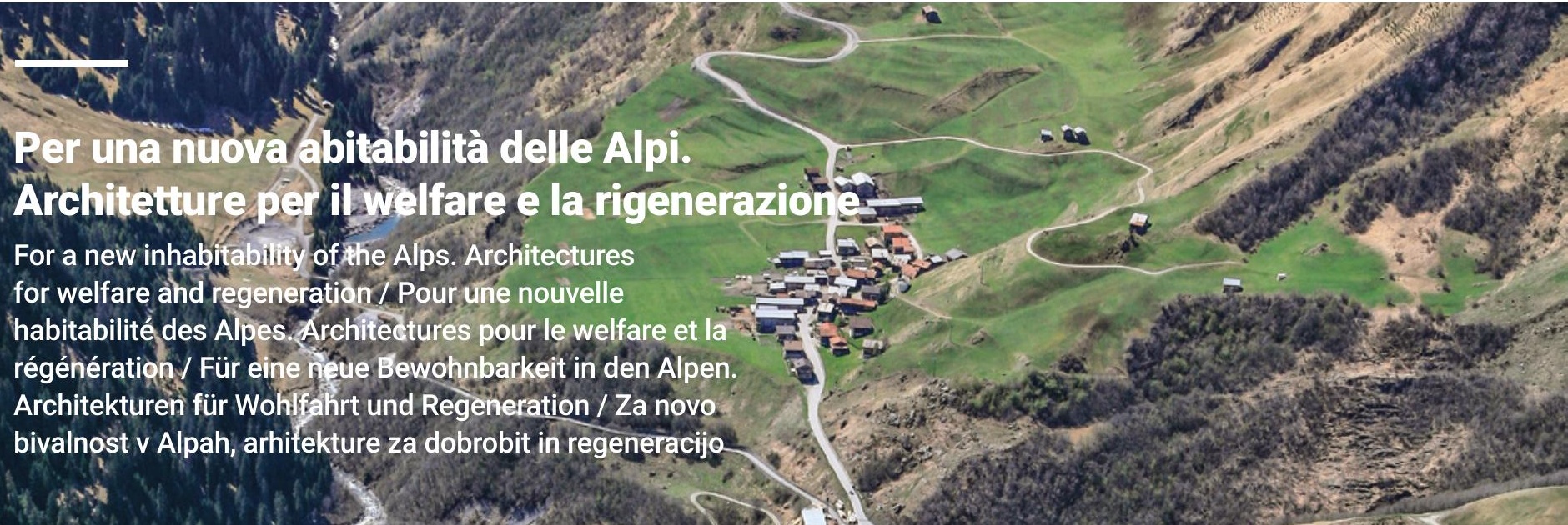
a $x \rightarrow 3$
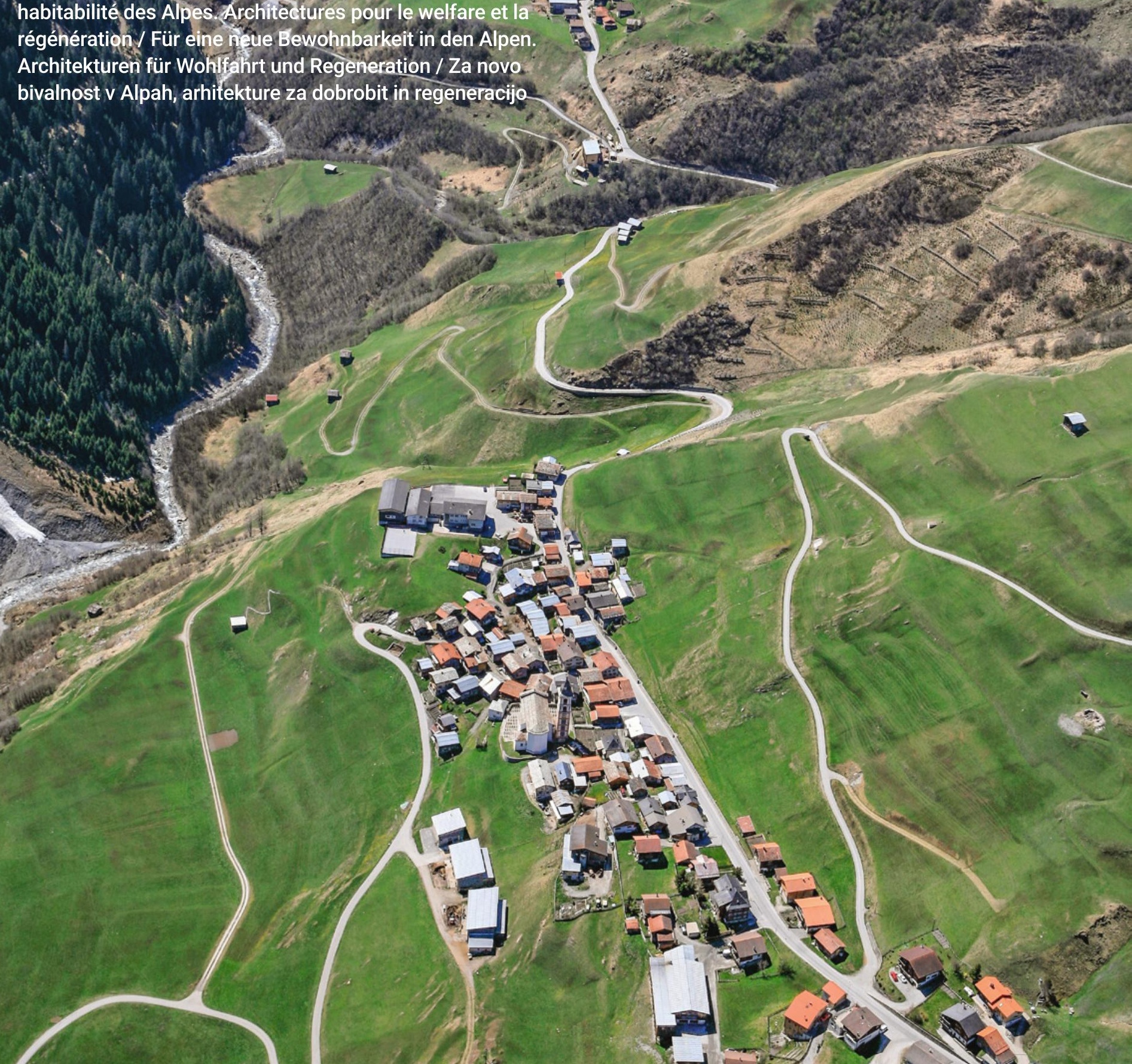


\section{Indice dei contenuti Contents}

\section{Temi}

Sulla centralità di spazio e territorio nel progetto di rigenerazione delle montagne e delle aree interne / On the centrality of space and territory in the project of regeneration of mountains and internal areas Antonio De Rossi, Laura Mascino

I servizi nelle Alpi italiane: quali e dove? Idee per uno scenario post-pandemico / Facilities in the Italian Alps: which ones and where? Ideas for a post-pandemic scenario

Giuseppe Dematteis

Manifesto di Camaldoli per una nuova centralità della montagna

Alla ricerca della distanza perduta. Rigenerare luoghi, persone e immaginari del riabitare alpino / In search of the lost distance. Regenerating places, people and images related to Alpine reinhabitation

Filippo Barbera, Andrea Membretti

Futuro e rigenerazione

Marco Bussone

\section{Esperienze}

Dorferneuerung zwischen Erhalten und Gestalten / 
Valades ousitanes, architettura e rigenerazione / Valades ousitanes,

Ostana e Topolò: hardware, software e welfare nelle comunità di "ritorno" / Ostana and Topolò: hardware, software and welfare in "return" communities

Margherita Valcanover

"Senza mostrare i muscoli": i progetti di rigenerazione di Tao+C e AZL nel solco della "prosperosa società" della Cina contemporanea / "Without flexing one's muscles": the regeneration projects of Tao+C and AZL in the wake of the "prosperous society" of contemporary China Edoardo Bruno, Dalila Tondo

L'archipel Butor. Une régénération, par la culture, d'un village soumis à la métropolisation genevoise / The Butor archipelago. A regeneration, through culture, of a village subject to the metropolisation of Geneva Arnaud Dutheil

Pratiche e progettualità di rigenerazione e welfare: il "Premio triennale Giulio Andreolli - Fare paesaggio" / Actions and projects of regeneration and welfare: the "Premio triennale Giulio Andreolli - Fare paesaggio" Giorgio Tecilla

Architetture e strategie per il welfare. Il caso di Brunico in Val Pusteria / Welfare architectures and strategies. The Bruneck case in Val Pusteria

Eleonora Gabbarini

Arhitektura oživlja / Architecture revives Kristina Dešman, Maja Ivanič

Si Crans-Montana meurt. Soigner le corps malade d'une station / If Crans-Montana dies. Taking care of the ailing body of a tourist resort Patrick Giromini 


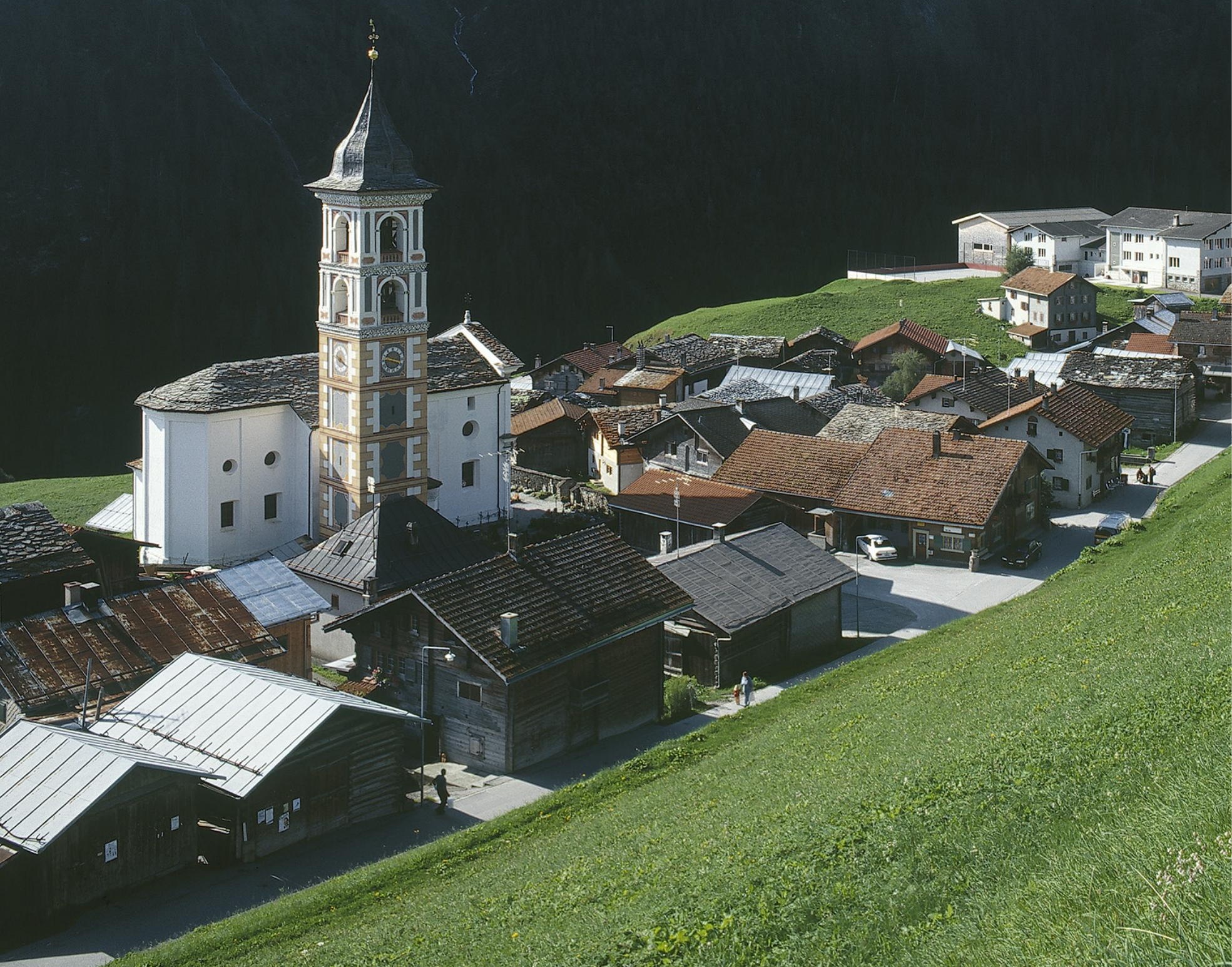




\title{
Dorferneuerung zwischen Erhalten und Gestalten
}

\author{
Il rinnovamento dei villaggi: tra conservazione e progettazione \\ The renovation of the villages: between conservation and design
}

\begin{abstract}
They are high-profile supporters of an architecture developed from existing structures, and both of them think of their work as a social task to a considerable degree: they are Armando Ruinelli (* 1954), who has been running an architectural office in Soglio (Bregaglia) since 1982, and Gion A. Caminada ( $\star 1957)$, who has been working as an architect, town planner and construction consultant in Vrin (Lumnezia) since the beginning of the 1990s. After their "years of apprenticeship" in Zurich, they both returned to their home villages, where not only did they carry out projects, but they also got involved in community issues. Initially, they both worked exclusively in their home valleys, but today they are known and sought after beyond their country's borders. The interest in the future of the peripheral Alpine valleys has remained a central aspect of their work.

A conversation about village regeneration, townscape protection and political commitment, recorded by Ludmila Seifert, Managing Director of the association Bündner Heimatschutz (Homeland Protection of the canton of Grisons).
\end{abstract}

\section{Armando Ruinelli}

Born in Soglio in 1954, he is an FAS architect. In 2000, he founded the studio Ruinelli Associati Architetti SIA with Fernando Giovanoli.

\section{Gion A. Caminada}

Born in Vrin (Switzerland) in 1957. He trained as a carpenter/joiner and attended the Kunstgewerbeschule (Arts and Crafts School). He graduated at the Swiss Federal Institute of Technology (ETH) in Zurich, and he then founded his own architectural office in Vrin. He has been professor of Architectural Design at the ETH Zurich since 1998.

\section{Ludmila Seifert}

Art historian, she has been Head of business of the association Bündner Heimatschutz (Homeland Protection of the Canton of Grisons) since 2011. In 2018, she received a recognition award from the canton of Grisons government for her work on the architectural history of the canton and her commitment to building culture issues as managing director of the association Bündner Heimatschutz.

Keywords

Caminada, Ruinelli, preservation, design, project, Vrin, Soglio, Switzerland 
Ludmila Seifert: Vor Jahren wurden prominente Stimmen laut, die eine weitgehende Vergandung der Berggebiete fordern - der Aufgabe der alten Bergbauerndörfer inklusive. Heute sprechen wir über Dorferneuerung, gehen also wie selbstverständlich davon aus, dass unsere Dörfer eine Existenzberechtigung haben. Warum soll man sie erhalten?

Armando Ruinelli: Ich sehe keinen grundsätzlichen Zwang zum Erhalt. Nur weil etwas existiert oder gar schön ist, heisst das noch nicht, dass es auch erhalten werden muss. Es braucht eine Funktion. Wir müssen uns von der Vorstellung einer traditionellen Dorfgemeinschaft mit einer Vielzahl von «einheimischen» Familien mit Kindern verabschieden. Es gibt zu wenig ortsansässige «Einheimische». Man muss sich anderen Ideen gegenüber öffnen. Rund 70\% der Schweizer Bevölkerung lebt in den Zentren - in Städten und darum herum, in der Agglomeration. Wir können den Städtern ein Refugium bieten, wo sie, als «Part-time-Einwohner» sozusagen, temporären Wohn- und Arbeitssitz nehmen können. Nicht nur ferienhalber, sondern für Wochen oder gar Monate. Ab und zu ziehen Leute auch tatsächlich von auswärts zu. Das sind oft besondere, interessante Persönlichkeiten mit einem kritischen Geist, die auch neue Sichtweisen einbringen, also auf ihre Weise zu einer Dorferneuerung beitragen. Aussteiger, Künstler. Für uns «Eingeborene» ist das Dorf Heimat, wir haben hier unsere Wurzeln, die uns das Leben im Dorf gut aushalten lassen. Aber für Auswärtige ist das zuweilen sehr schwierig. Wir müssen ihnen helfen, sich zu integrieren, was allerdings ein gegenseitiges Verständnis bedingt. In den Bergtälern herrscht oft ein Widerwille, sich dem Fremden gegenüber zu öffnen, in der Meinung, man wisse schon selber, was gut für einen ist. Aber das ist nur bedingt richtig. Einflüsse von aussen sind bereichernd, sie helfen der Regeneration. Man kann ja nur wissen, was man selber will, wenn man sich auch mit dem «Anderen» auseinandersetzt.

Vorherige Seite / In apertura Vrin, Graubünden / Vrin, Grigioni (foto Gion A. Caminada)
Gion A. Caminada: Die Gründe für die Erhaltung der Dörfer gilt es immer wieder neu zu definieren. Vor dreissig Jahren haben wir in Vrin versucht, das 500-Seelen-Modell von Peter Rie-
Ludmila Seifert: Qualche anno fa, molte voci di spicco hanno chiesto sempre più insistentemente la progressiva riforestazione delle aree montane che prevedesse anche l'abbandono dei villaggi tradizionali abitati dai contadini di montagna. Oggi si parla di rinnovare questi villaggi, dando quindi naturalmente per scontato che essi abbiano ancora il diritto di esistere. Perché dovrebbero essere conservati?

Armando Ruinelli: Io non credo vi sia l'obbligo di conservare i villaggi. Solo perché qualcosa esiste o è bello non significa che debba essere conservato. Ha bisogno anche di una funzione. Dobbiamo abbandonare l'idea di una tradizionale comunità di villaggio costituita da un gran numero di famiglie "del posto" con bambini. In questi villaggi oggi ci sono troppo pochi "nativi", bisogna aprirsi a nuovi scenari. Circa il $70 \%$ della popolazione svizzera vive nei centri urbani - nelle città e nelle loro immediate vicinanze, nell'agglomerato urbano. Agli abitanti delle città possiamo offrire un rifugio in cui possano trovare un luogo di lavoro e di vita temporaneo, in quanto "residenti part-time", per così dire. Non solo per le vacanze, ma per settimane, o addirittura mesi. Ogni tanto nei villaggi si trasferiscono persone provenienti perfino da altre città. Si tratta spesso di personalità particolari e interessanti, dotate di spirito critico, spesso anticonformisti, artisti, che portano con sé anche nuove prospettive, contribuendo così a modo loro al rinnovamento del villaggio. Per noi "nativi" il villaggio è la nostra casa, qui abbiamo le nostre radici, il che rende vivere in questi luoghi più tollerabile. Ma a volte è molto difficile per chi viene da fuori. Dobbiamo aiutare queste persone a integrarsi, il che richiede, tuttavia, una comprensione reciproca. Nelle valli montane c'è spesso una certa riluttanza ad aprirsi agli estranei, nella convinzione di sapere già cosa sia meglio per se stessi, ma questo è vero solo in parte. Le influenze esterne ci arricchiscono, favoriscono la rigenerazione. Possiamo sapere davvero che cosa vogliamo solo se ci confrontiamo anche con "l'altro".

Gion A. Caminada: Le ragioni per la conservazione dei villaggi devono essere continuamente ridefinite. Trent'anni fa, a Vrin, abbiamo cercato di attuare il modello di 500 persone di Peter Rieder, 
der umzusetzen, welches das Funktionieren eines Dorfes von einer bestimmten Mindest-Bevölkerungsanzahl abhängig macht. Das Ziel wurde nicht erreicht. Dieses Scheitern hat bei mir zu einer radikal anderen Sicht geführt. Ich empfinde es als Privileg, an einem Ort zu wohnen, in dem fast niemand wohnen will. Statt von Quantitäten sollten wir anfangen, von Qualitäten zu sprechen. Qualität in Bezug auf eine bestimmte Intention. Ich denke, es braucht, unabhängig von der Grösse, massgeschneiderte Ideen für ein Dorf. Ideen, mit denen

Abb. 1 / Fig. 1

Stallumnutzung in Soglio, Graubünden

/ Riqualificazione

stalla a Soglio,

Grigioni (foto (c)

Armando Ruinelli) sich die Leute identifizieren, an denen sie teilhaben, die sie zelebrieren können. Das generiert Lebensqualität. Man muss die Möglichkeiten einer kleinen Gemeinschaft ausloten.

LS: Aber das Rieder'sche Modell war ja auch ein ökonomisches Modell, ausgehend von der Frage, secondo cui il funzionamento di un villaggio dipende da un numero minimo di abitanti. L'obiettivo di questo modello non è stato raggiunto, e il suo fallimento mi ha portato a sviluppare una visione completamente diversa della questione. Considero un privilegio poter vivere in un posto dove quasi nessuno vuole abitare. Invece che di quantità, dovremmo iniziare a parlare di qualità, in relazione a un'intenzione specifica. Credo che, indipendentemente dalle dimensioni, in un villaggio ci sia bisogno di idee su misura per le sue esigenze. Idee in cui le persone si identifichino, a cui prendano parte, che possano celebrare. Questo genera qualità della vita, e perciò è necessario esplorare le possibilità offerte da una piccola comunità.

LS: Ma il modello di Rieder era anche un modello economico, basato sulla questione fondamentale
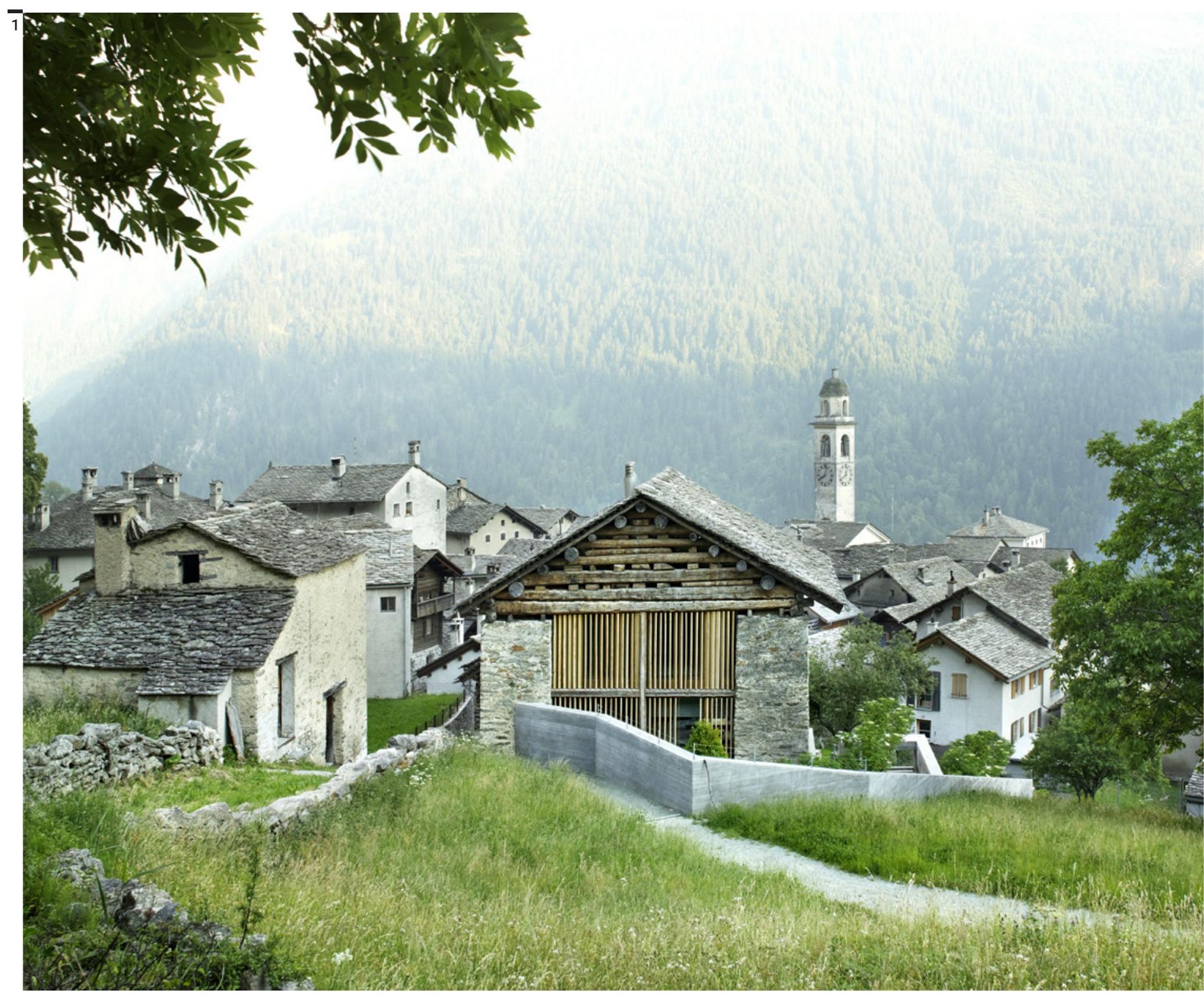


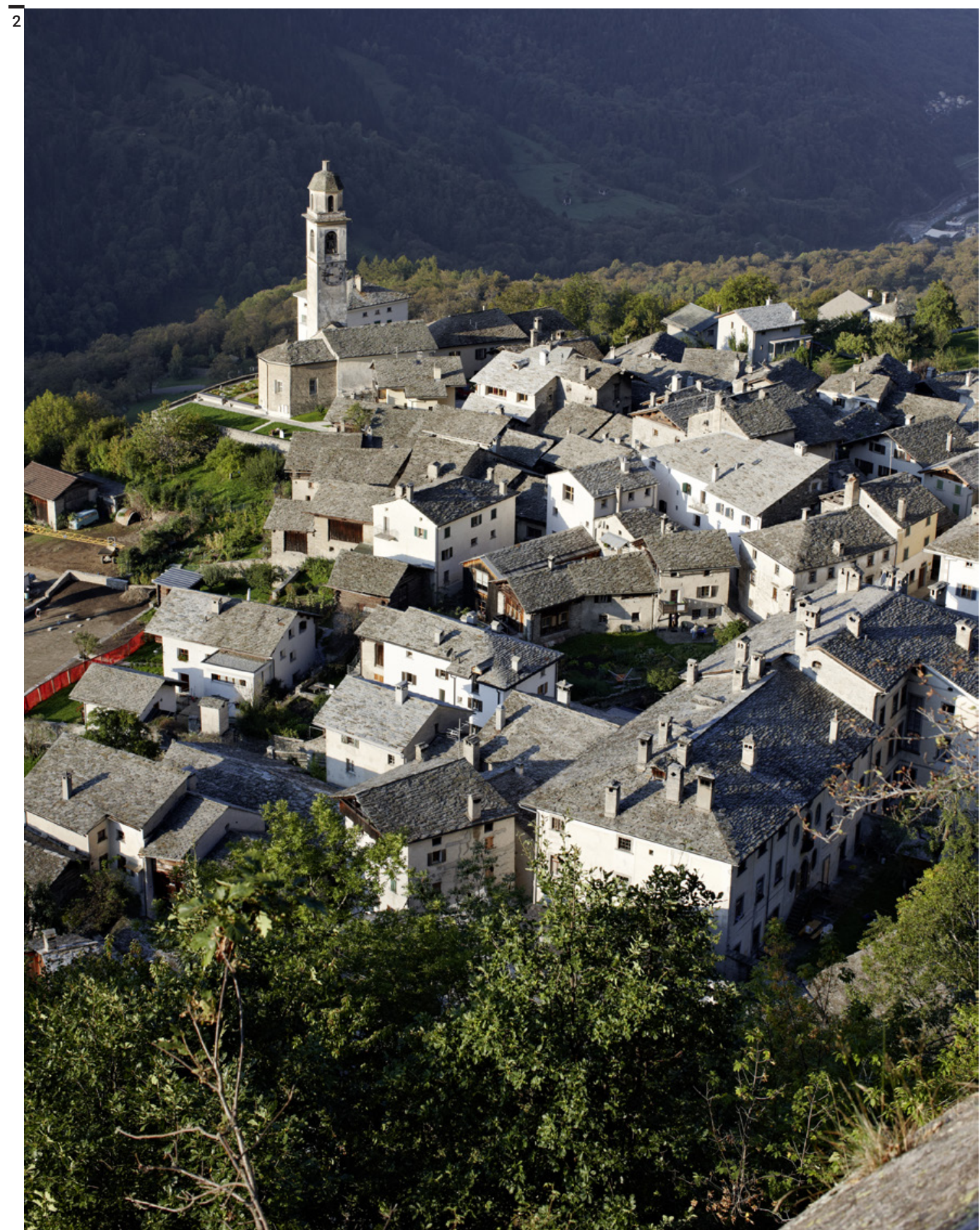

Abb. 2 / Fig. 2 Aussicht von Soglio,

Graubünden /

Veduta di Soglio,

Grigioni (foto Ralph

Feiner). unter welchen Bedingungen ein Dorf wirtschaftlich überleben kann.

GC: Ja, natürlich, aber da haben die Gemeindefusionen eine Erleichterung gebracht. Ich war ursprünglich gegen den Zusammenschluss. Ich hatte den Verdacht einer aufkommenden Verantwortungslosigkeit gegenüber dem, was wir in den letzten Jahrzehnten erarbeitet hatten. Jetzt, da die Fusion erfolgt ist, sehe ich durchaus Vorteile. Die delle condizioni in cui un villaggio può essere economicamente sostenibile.

GC: Sì, certo, ma a tal proposito le fusioni tra comuni hanno portato un po' di sollievo. Inizialmente ero contrario alla fusione, mi preoccupava la crescente mancanza di responsabilità verso quello che abbiamo progettato nel corso degli ultimi decenni. Ora che la fusione è avvenuta, ne vedo anche i vantaggi. Nelle piccole comunità di un tempo le diffi- 
ehemaligen Kleingemeinden wurden finanziell entlastet. Vor der Fusion wollten viele Vriner ihr Wasser unbedingt ins Kraftwerk nach Vals überleiten, um von den Wasserzinsen zu profitieren. Heute ist das kein Thema mehr. Ohne das ökonomische Damoklesschwert im Nacken ist man freier und kann andere Möglichkeiten, die für das gute Lebensgefühl des Dorfes wichtig sind, in Betracht ziehen.

AR: Ich war auch lange gegen die Fusion. Aber den kleinen Gemeinden hat man die Last genommen, eigenständig funktionieren zu müssen. Es geht nicht nur um die Finanzen, sondern auch um die Politik, um die Besetzung des Gemeinderats oder des Gemeindepräsidiums zum Beispiel. Das war am Schluss ganz schwierig.

GC: Seit der Fusion achtet man auch ein bisschen mehr auf die Unterschiede zwischen den einzelnen Dörfern. Da gilt es anzusetzen: die Differenzen herauszuschälen und diese zu stärken. Was ist zum Beispiel in Vrin möglich, was in Morissen nicht möglich ist - und umgekehrt. Wenn man auf den spezifischen Ressourcen und Qualitäten des jeweiligen Dorfes aufbaut, wirkt sich das positiv auf die ganze Gemeinde aus.

AR: Dabei sollte man unbedingt verhindern, dass Monokulturen entstehen. Nehmen wir das Beispiel der Kunstgalerien oder Gesundheitszentren, die da und dort ein Dorf quasi «besetzen». So interessant diese Ansätze auch sein mögen, es besteht da immer die Gefahr der Monopolisierung. Als Einheimischer ist man dann entweder im Dunstkreis dieses Betriebs - oder man ist weg vom Fenster. In Soglio hat man bisher nur die etwas einfacheren Häuser und die Ställe an Auswärtige verkauft, sozusagen die «Plastikbecher». Jetzt aber steht das «Tafelsilber» zur Disposition, die herrschaftlichen Palazzi der Familie von Salis. Gerade eben wurde eines veräussert und das wird jetzt aufwändig umgebaut glücklicherweise scheint da etwas zu passieren, das auch für das Dorf interessant werden könnte. Aber stellen wir uns vor, da kommt eine auswärtige Galerie oder von mir aus, eine Klinik und buttert in die restlichen Palazzi Millionen rein. Dann zählt da nur noch die Kunst oder der Gesundheits «tourismus» - und der Rest ist nichts mehr wert.

LS: Beim Erneuern stellt sich ja immer auch die Frage nach dem Umgang mit dem historischen Bestand. Wie ist Euer Verhältnis zum Ortsbildschutz?

GC: Der Gedanke des «Erhalts» ist für das Bergdorf relativ neu. Der Bergbauer hat immer die Überwindung des Hier und Jetzt gesucht, er war immer bestrebt, seine - grundsätzlich als ungenügend empfundene - Situation zu verbessern. Diese Mentalität hat sich nicht geändert. Der Vriner will nach wie vor nicht nur erhalten. Das wollen coltà economiche si sono alleviate. Prima della fusione, molti cittadini a Vrin volevano fare deviare la propria acqua verso la centrale idroelettrica di Vals per poter beneficiare delle imposte sul consumo idrico. Oggi non succede più. Senza la spada di Damocle delle finanze sul capo, si è più liberi e si possono prendere in considerazione altre opzioni, che sono importanti per raggiungere un buono stile di vita nel villaggio.

AR: Anch'io sono stato a lungo contrario alla fusione, ma in effetti le piccole comunità sono state sollevate dall'onere di dover funzionare in modo indipendente. Non è solo una questione economica, ma anche politica, come la nomina del consiglio comunale o del comitato direttivo locale. Alla fine, è stato un processo molto complicato.

GG: Da quando c'è stata la fusione dei comuni, le persone prestano anche maggiore attenzione alle differenze tra i singoli villaggi. È da qui che dobbiamo iniziare: bisogna identificare le differenze e rafforzarle. Ad esempio, ciò che è possibile a Vrin non lo è a Morissen - e viceversa. Se si sfruttano le risorse e le qualità specifiche del singolo villaggio si ottengono effetti positivi sull'intera comunità.

AR: $\mathrm{Al}$ contempo, è assolutamente necessario impedire lo sviluppo di monoculture. Prendiamo ad esempio le gallerie d'arte o le cliniche, che "occupano" diversi villaggi. Per quanto questi approcci possano essere interessanti, esiste sempre il rischio della monopolizzazione. In quanto residente locale, se non si fa parte della sfera di influenza di questi business si è fuori dai giochi. A Soglio, finora, solo le case più semplici e le stalle sono state vendute a terzi, le "briciole", per così dire. Ora c'è in palio "il piatto forte", i maestosi palazzi della famiglia von Salis. Uno è appena stato venduto e in questo momento è in fase di estesa riconversione - per fortuna, sembra che stia accadendo qualcosa che potrebbe essere interessante per l'intero villaggio. Ma proviamo ad immaginare l'arrivo di una galleria d'arte esterna o, per quanto mi riguarda, anche di una clinica, che investe milioni negli altri palazzi. Allora conterà solo il "turismo" dell'arte o della salute, e il resto non avrà più alcun valore.

LS: Quando si rinnova, c'è sempre la questione di come gestire il patrimonio edilizio esistente. Qual è il vostro rapporto con la tutela del paesaggio abitato locale?

GG: L'idea di "conservare" è relativamente nuova per il villaggio montano. Il contadino di montagna ha sempre cercato di affrontare il qui e ora, provando continuamente a migliorare la propria condizione, che è sempre stata percepita come fondamentalmente insoddisfacente. Questa mentalità non è cambiata. L'abitante di Vrin non vuole solo conser- 
einzig jene, die in einer gewissen Distanz zu den Dingen stehen, die quasi «von aussen» einen Verlust realisieren und aus dieser Empfindung heraus Gegensteuer geben. Der Wunsch nach Überwindung dessen, was man hat im Hinblick auf ein imaginiertes Paradies, einen Sehnsuchtsraum finde ich als Geisteshaltung spannend und unter den neuen Wirklichkeiten herausfordernd. Für mich stellt sich die Frage: Wie können wir das «Schützen» im strengen, denkmalpflegerischen Sinn und das unreflektierte «Weiterentwickeln» um jeden Preis vereinen zu etwas Neuem, das den Bestand respektiert und kontinuierlich weiterbaut.

AR: Ich denke auch, dass das Streben nach Veränderung im Sinne von Verbesserung der menschlichen Psyche eigen ist - dass sich das (vermeintlich) Bessere dann häufig nicht unbedingt als solches erweist, ist eine andere Sache. Aber den Erneuerungswillen braucht es unbedingt. Das museale Bewahren ist kein zukunftsträchtiger Ansatz. Sonst haben wir eben die zwei Situationen, die Städte, die sich erneuern, gesellschaftlich, politisch und auch architektonisch, und die Dörfer, die im status quo verharren bis zur Leblosigkeit.

GG: Aber diese Haltung schliesst ja das Erhalten überhaupt nicht aus. Uns beiden geht es ja immer auch um das Ausloten des Potenzials dessen, was da ist. Man darf den Schutzgedanken einfach nicht zu eng fassen. Heute zelebriert man die Geschichte, weil man sie schön und interessanter findet. Aber bedeutet sie uns wirklich etwas? So bringt man zum Beispiel religiöse Wandmalereien, die zu einem bestimmten Zeitpunkt aus einer bestimmten religiösen Haltung heraus zugedeckt worden sind, wieder zum Vorschein, ohne dass man dazu einen ernsthaften Bezug hätte - sozusagen als Dekoration. Das macht wenig Sinn. Wenn ich an einem bedeutenden historischen Haus arbeite, erlebe ich immer wieder, wie mutlos sich die Behörden geben, wenn es darum geht, prägnante Eingriffe zuzulassen - ja überhaupt zu beurteilen. Da wird mit dem Hinweis auf den Denkmalschutz schnell mal einfach alles verboten. Das ist mir zu simpel. So entzieht man sich der kritischen Auseinandersetzung.

LS: Das ist aber ja verständlich. Nicht jeder ist wirklich begabt im Weiterbauen am Bestand. Die wenigsten Eingriffe sind sorgfältig durchdacht und sensibel gemacht. Da ist es einfacher, man stellt verbindliche Regeln auf, die dann halt für alle gelten. Vom ästhetischen, baukulturellen Standpunkt aus hatte die Mangelwirtschaft der Vergangenheit ja ihre Vorteile. Die ökonomisch und auch gesellschaftlich bedingte Reduktion von Material und Form, die unbedingte Notwendigkeit, mit der To- vare. Solo chi prova un certo distacco dalle cose vuole farlo, chi percepisce una perdita, ma "dall'esterno", per così dire, e basandosi su questo sentimento, prende le dovute precauzioni. Il desiderio di migliorare ciò che si ha, con la prospettiva di raggiungere una sorta di paradiso immaginario, uno spazio verso cui tendere, mi sembra uno stato d'animo interessante e stimolante nel contesto di queste nuove realtà. La domanda che mi sorge spontanea è: come possiamo conciliare la "protezione" in senso stretto, in un'ottica di conservazione del patrimonio architettonico, e lo "sviluppo continuo", spontaneo e a ogni costo, con qualcosa di nuovo, che rispetti l'esistente e allo stesso tempo lo sviluppi costantemente? AR: Penso anch'io che la ricerca del cambiamento, inteso come miglioramento, appartenga naturalmente alla mente umana. Che ciò che appare presumibilmente migliore finisca poi, in molti casi, per non esserlo, è un'altra questione, ma la volontà di rinnovamento è assolutamente necessaria. La conservazione in stile museale non è un approccio promettente per il futuro. Si creerebbero altrimenti due situazioni distinte: da una parte le città si rinnoverebbero dal punto di vista sociale, politico e architettonico, mentre dall'altra i villaggi resterebbero bloccati nello status quo, fino a scomparire.

GG: Ma questo atteggiamento non preclude affatto la conservazione. Entrambi cerchiamo costantemente di esplorare il potenziale di ciò che già c'è, semplicemente non bisognerebbe restringere troppo il concetto di conservazione. Oggi celebriamo la storia perché la troviamo bella, interessante, ma significa davvero qualcosa per noi? Ad esempio, i dipinti murali religiosi che erano stati coperti in un determinato periodo storico per volere di una certa posizione religiosa, oggi vengono riportati alla luce, senza che esista però nessun legame reale tra noi ed essi - a scopo puramente decorativo, per così dire. Non ha molto senso. Quando lavoro su un'abitazione storica di valore mi rendo costantemente conto di quanto poco coraggio mostrino le istituzioni quando si tratta di approvare, o anche solo di prendere in considerazione, interventi significativi. In questi casi, con la scusa della conservazione dei monumenti, viene semplicemente proibita qualsiasi cosa. Così è troppo semplice, secondo me. In questo modo si evita la discussione critica.

LS: Beh, però è comprensibile. Non tutti sono davvero portati a lavorare su edifici storici e, del resto pochissimi interventi sono pensati minuziosamente e realizzati con criterio. Perciò è più facile imporre norme vincolanti applicabili a tutti i casi. Dal punto di vista estetico e della cultura edilizia, la carenza di risorse economiche del passato aveva i suoi vantaggi. La carenza di materiali e forme, dovuta alle condizioni economiche e sociali, e la stringente necessità di lavorare con la topografia e non con- 


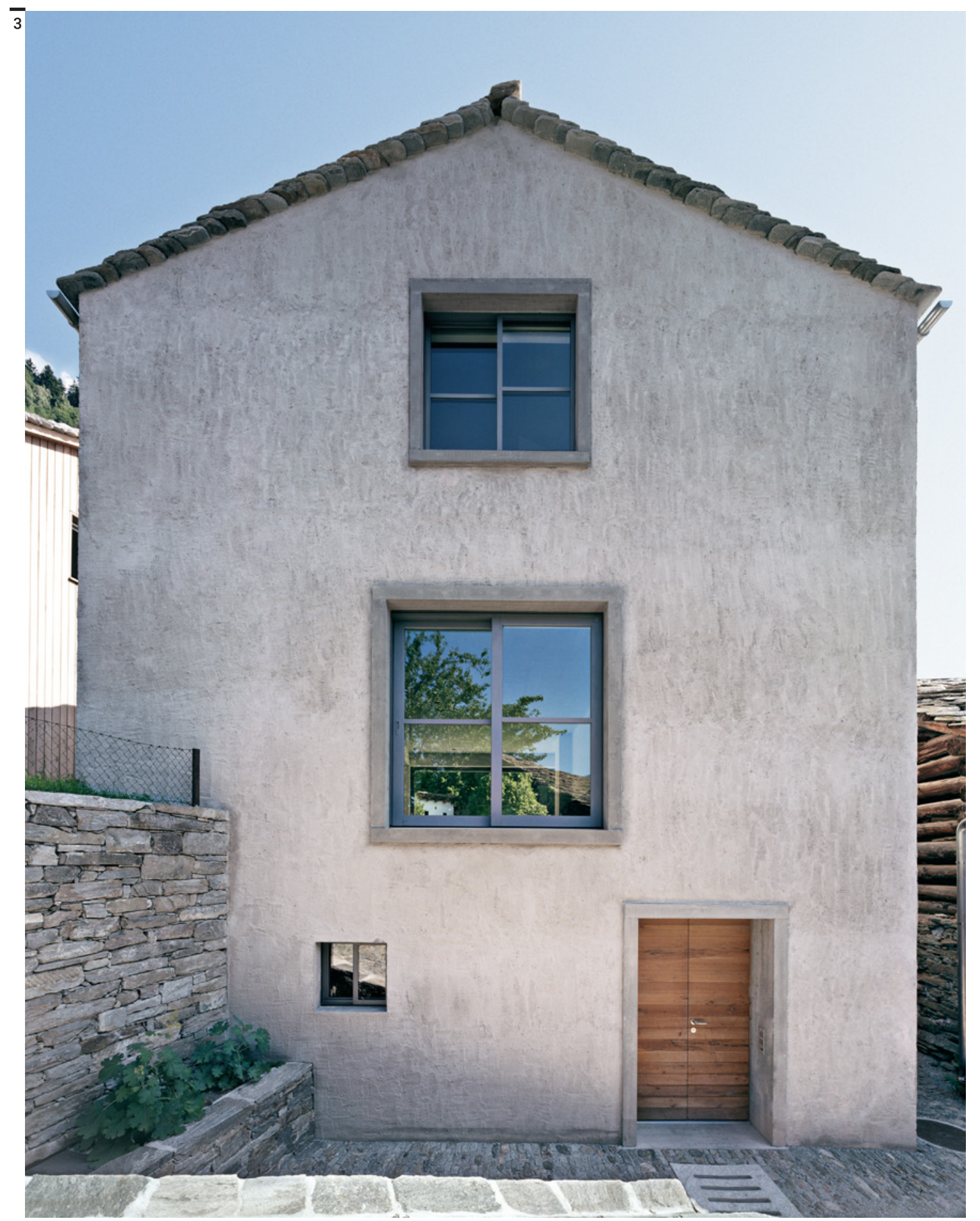

Abb. 3 / Fig. 3

Atelierhaus in Soglio, Graubünden, Ruinelli Associati AG Architetti SIA

(Schweizerischer Ingenieur- und Architektenverein)

/ Casa Atelier a

Soglio, Grigioni, Ruinelli Associati Architetti (foto (C) Armando Ruinelli). pografie zu arbeiten und nicht gegen sie - das alles hat Ortsbilder hervorgebracht, die wir heute als charismatisch, als kraftvoll, als stark empfinden. Heute hingegen ist überall alles möglich. Dieses Unspezifische hat dem Gesicht unserer Dörfer nicht unbedingt gutgetan. Da geht es um die grundsätzliche Frage: Wie bringt man Ortsbildschutz und Ortserneuerung zusammen, wie findet man einen Ausgleich zwischen Bewahren und Ge- tro di essa, hanno permesso la creazione dei paesaggi abitati locali che oggi consideriamo carismatici, dotati di forza e di grande potenziale. Oggi, al contrario, tutto è possibile ovunque e questa mancanza di specificità non ha sempre fatto bene all'estetica dei nostri villaggi. L'interrogativo fondamentale è: come possiamo coniugare la tutela dell'immagine del paesaggio abitato con il rinnovamento del luogo, trovare un equilibrio tra conservazione e progetta- 


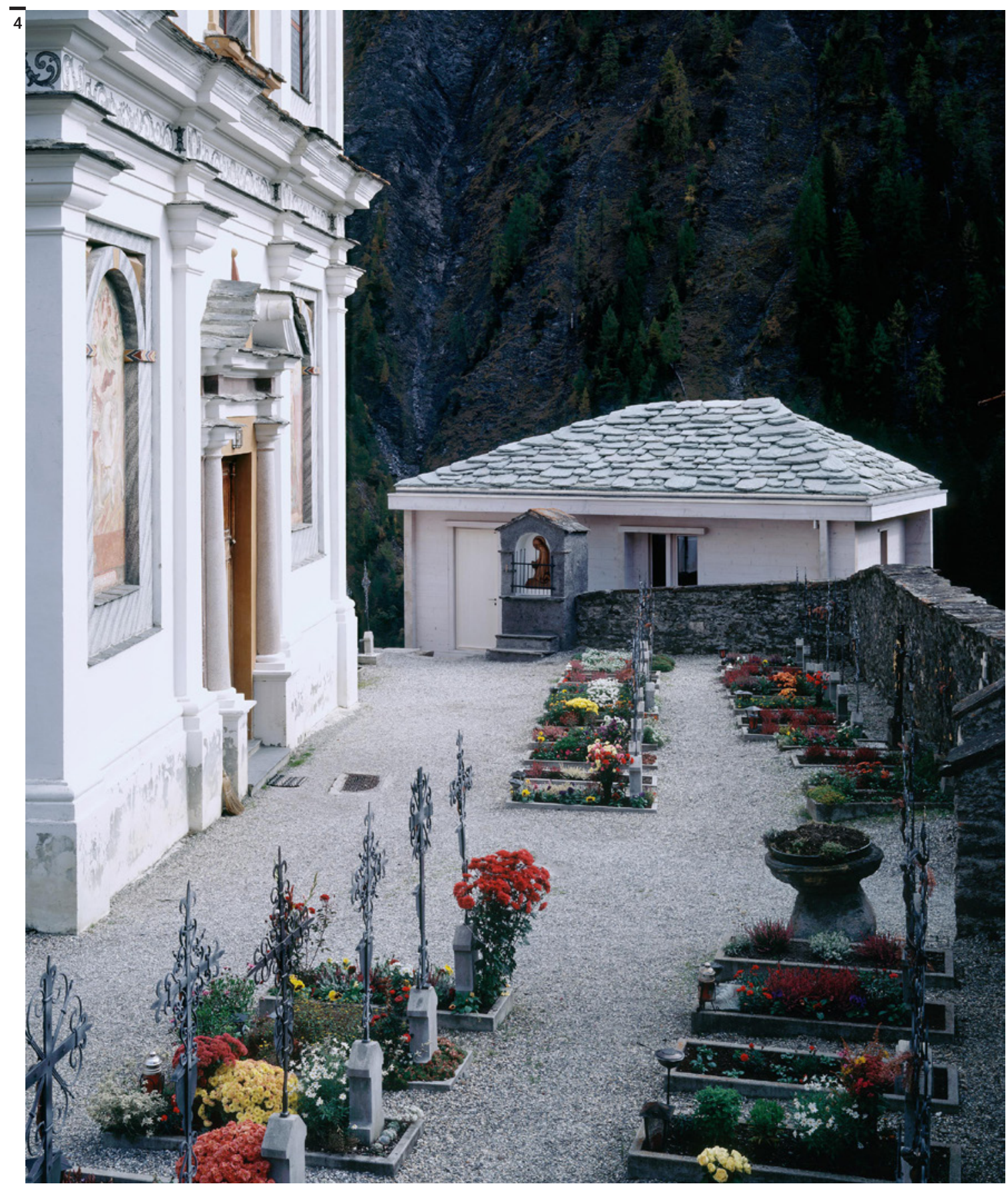

Abb. 4 / Fig. 4

Totenstube (Stiva da morts), Vrin, Graubünden, Gion

A. Caminada / Stiva da morts, Vrin, Grigioni, Gion A. Caminada (foto Lucia Degonda). stalten? Schutzbestimmungen sind ja nur der Versuch, die potentiell zerstörerischen Auswirkungen der Eingriffe einzudämmen.

GC: Ja, eine Mindestgesetzgebung braucht es natürlich...

AR: ... aber Gesetze können eben auch Gutes verhindern. Erneuerung hat auch mit zeitgenössischer Architektur zu tun. Ich meine, dass man viel mehr differenzieren sollte. Es gibt Sachen, die man unbedingt erhalten muss, aber es gibt auch in historischen Dörfern Situationen, die man verändern können sollte. Wichtig ist natürlich, dass das, was zione? Dopotutto, le norme sulla conservazione non sono altro che un tentativo di contenere gli effetti potenzialmente distruttivi degli interventi.

GC: Sì, ovviamente una legislazione minima è necessaria...

AR: ...ma le leggi possono anche ostacolare cose buone. Il rinnovamento dovrebbe avere a che fare anche con l'architettura contemporanea. Penso che si dovrebbe operare una distinzione molto più netta: ci sono cose che è assolutamente necessario preservare, ma anche situazioni, nei centri storici, che dovrebbero poter essere modificate. Sarebbe anche 
an die Stelle des Alten tritt, mindestens ebenso gut, womöglich besser ist. Und dafür, da gebe ich Dir Recht, besteht keine Garantie. In der Regel wird es schlechter. Das Problem ist, dass das Bauen zur Investition geworden ist. Früher war es eine Notwendigkeit. Sobald Geld ins Spiel kommt, rutscht Dir das aus der Hand. Das kann sich katastrophal auswirken.

LS: Gibt es Mittel, Qualität zu gewährleisten?

AR: Ich denke da vor allem an das Instrument der Gestaltungsberatung durch einen Gestaltungsbeirat aus auswärtigen Fachleuten, die unabhängig von persönlichen Verflechtungen etwas sachlich beurteilen und Qualität einfordern können. Langfristig bewährt sich das, auch wenn natürlich auch nur punktuell interveniert werden kann.

GC: Auch mit Quartierplänen kann man viel erreichen. Aber die Sache ist hochkomplex und Regulierungen haben ihre Grenzen. Die Hoffnung ist, dass man gut gebildete Leute mit einer klaren Geisteshaltung hat, die bereit sind, sich einzumischen. In Vrin, da haben wir damals das Baugesetz neu geschrieben. Dieses konnte nach der Gemeindefusion nicht in das übergeordnete kommunale Baugesetz integriert werden. Nun werden uns die Baugesuche zur Beurteilung vorgelegt und so können wir das Baugeschehen direkt beeinflussen. Aber so was passiert nicht von allein, das braucht konstantes Engagement - über Jahre hinweg. Man muss mit den Einwohnern, den Behörden und den politischen Amtsträgern in einen ernst gemeinten Dialog treten und mit ihnen argumentieren. Dann kann man etwas bewirken. In Graubünden haben wir einige ausserordentlich gute Architekten. Das Problem ist nur, dass sich die meisten von ihnen nicht exponieren und politisch engagieren. Sie sind mehr oder weniger auf ihre Objekte fixiert, wollen «schöne» Häuser bauen. Das ist schon einmal sehr gut. Auch die Mehrzahl der abgehenden Architekten der ETH, wo ich lehre, sind fähig, schöne Dinge zu entwerfen. Das ist aber zu wenig und führt noch zu keinem guten Ort. Ich denke, wir müssen den Begriff «Schönheit» um weitere Komponente ergänzen. Schönheit liegt tief unter der Oberfläche. Aber zurück zu Deiner Frage: Ich denke, mit ehrlich gemeintem, persönlichem Engagement lässt sich etwas bewirken.

AR: Was Du ansprichst ist das Monte-Carasso-Modell von Luigi Snozzi. Es braucht vor Ort eine Persönlichkeit - eine Art «despota illuminato» - der sagt, wo's langgeht. Da und dort sind auf diese Weise interessante Geschichten entstanden - politisch, städtebaulich, architektonisch. Daneben wird an den meisten Orten einfach vor sich hingebaut. Auf eine gewisse Weise sind wir auch privilegiert, dass man uns überhaupt einbezieht und gewisse Prozesse mitgestalten lässt. Das hängt immer zusammen importante, ovviamente, che ciò che prende il posto del vecchio fosse almeno di pari valore e, se possibile, migliore di quest'ultimo. E in questo sono d'accordo con te, non ci sono garanzie, di solito il nuovo risulta peggiore. Il problema è che costruire è diventato un investimento, mentre una volta era una necessità. Quando entra in gioco il denaro, sfugge tutto di mano e le conseguenze possono essere catastrofiche. LS: Esistono mezzi per garantire la qualità?

AR: Mi viene in mente principalmente lo strumento della consulenza progettuale da parte di un comitato consultivo di progettazione composto da esperti esterni, che possano esprimere valutazioni obiettive ed esigere qualità nella progettazione, senza l'influenza di condizionamenti personali. A lungo termine, questo può portare a buoni risultati, anche se, ovviamente, si può intervenire solo in modo puntuale.

GC: Anche con i piani di vicinato si può ottenere molto, ma la questione è molto complessa e le normative hanno i loro limiti. La speranza è che ci siano persone ben istruite, con una mentalità aperta e pronte a mettersi in gioco. A Vrin, all'epoca, abbiamo scritto la nuova normativa edilizia. Tuttavia, dopo la fusione delle comunità, non è stato possibile integrarla alla legge sull'edilizia comunale di livello superiore. Oggi ci vengono sottoposte richieste di costruzione per una valutazione, quindi possiamo influenzare direttamente il processo di progettazione, ma tutto questo non succede per caso, richiede uno sforzo costante nel corso degli anni. Bisogna avviare un dialogo serio con i residenti, le autorità e i funzionari politici, solo questo può fare la differenza. Ci sono alcuni architetti davvero bravi nei Grigioni. Il problema è che molti di loro non si espongono e non sono politicamente impegnati. Sono tutti più o meno concentrati sui loro progetti, vogliono costruire case "belle" e questo è già un punto di partenza molto positivo. La maggior parte degli architetti che escono dall'ETH di Zurigo, dove insegno, è in grado di progettare cose belle, ma questo non è ancora abbastanza e non porta da nessuna parte. Penso sia necessario arricchire la definizione di "bellezza" di altri attributi, la vera bellezza si trova molto sotto la superficie. Tornando alla tua domanda: penso che con un coinvolgimento personale profondamente sincero si possano ottenere risultati.

AR: Ti riferisci al modello Monte Carasso di Luigi Snozzi. C'è bisogno di una personalità in loco, di una sorta di "despota illuminato", che sappia in che direzione andare. In questo modo qua e là sono successe cose interessanti - dal punto di vista politico, in termini di pianificazione urbana e a livello architettonico. Allo stesso tempo, nella maggior parte dei villaggi si continua semplicemente a costruire. In un certo senso, abbiamo il privilegio di essere perlomeno coinvolti e di poter prendere parte ad alcuni processi. In que- 
mit ein paar wenigen Leuten, die sagen: Wir probieren das mal ein bisschen anders.

LS: Dann hat man ein paar Orte, die das Glück haben, solche Leute zu haben und die andern wursteln beliebig vor sich hin.

GC: Ja, aber wir können auch ausserhalb unseres eigenen Dorfes wirken, wenn wir in fremde Situationen eintauchen und versuchen, diese zu verstehen. Voraussetzung ist natürlich, dass man ein Gegenüber hat, das initiativ und offen ist. In Valendas zum Beispiel, da gab es eine Gruppierung, die das vor sich hin dümpelnde Dorf unbedingt weiterbringen wollte - und da haben neben mir viele Auswärtige mitgemacht, um das in gute Bahnen zu lenken. Die Initiative von innen mit der Intervention von aussen, das ist eine vielversprechende Mischung. In Disentis, wo ich für das Benediktiner-Kloster gearbeitet habe, konnte ich auch Ideen hineinbringen, die über den Einzelbau hinausgehen. Im letzten Semester habe ich zusammen mit meinen Studenten einige Überlegungen zur anstehenden Ortsplanung der fusionierten Gemeinde Ilanz entwickelt - da hatten wir als Gegenüber eine sensitive Gemeindepräsidentin, die selber ausgebildete Architektin ist. Es ging darum, für die einzelnen Fraktionen der fusionierten Grossgemeinde unterschiedliche Ideen herauszuarbeiten. Ideen entstehen über die Wahrnehmung, das ist nichts Abstraktes, das man losgelöst vom Kontext einfach hineinschmeissen kann. Man muss erkennen, was vorhanden ist und erspüren, was sich daraus entwickeln könnte. Sich einbringen zu wollen, verlangt von einem selber eine ehrliche Grundhaltung. Ich muss am Anfang mit Bestimmtheit sagen können: Ich habe die Leute gerne, ich respektiere die Differenzen, die unterschiedlichen Sichtweisen. Damit muss man zurechtkommen. Man kann nicht von aussen kommen und den Menschen vor Ort wie ein Missionar die eigenen Ideen überstülpen. Dann mögen Dich die Leute nicht. Und Du bist auf verlorenem Posten.

LS: Seit Anfang 2016, seit der Einführung des Zweitwohnungsgesetzes, haben wir in Graubünden eine neue Situation, die es nicht unbedingt leichter macht, Entwicklungsperspektiven aus einer ideellen oder philosophischen Grundhaltung heraus zu entwickeln. Das Gesetz verbietet Gemeinden mit mehr als 20\% Zweitwohnungen - das sind in Graubünden die meisten - weitere Zweitwohnungen zu bauen. Um die Auswirkungen im Berggebiet etwas abzuschwächen, wurden unter Vorschiebung von Ortsbildschutz-Interessen verschiedene Ausnahmen geschaffen. So dürfen historische Bauten, die altrechtlich nicht als Wohnbauten dienten, in Zweitwohnungen umgenutzt werden, wenn sie «ortsbildprägend» sind und ihr Erhalt anders nicht gesichert werden kann. Als «ortsbildprägend» gelten Gebäude, «die durch ihre Lage und Gestalt we- sti casi, si tratta sempre di poche persone che dicono: "proviamo a fare le cose in modo un po' diverso". LS: Ci sono alcuni luoghi che hanno la fortuna di avere persone simili, mentre altri vanno semplicemente avanti senza un piano preciso.

GG: Sì, però possiamo fare qualcosa anche al di fuori dei nostri villaggi, quando ci troviamo in situazioni diverse e proviamo a comprenderle. Il prerequisito, naturalmente, è la presenza di un interlocutore proattivo e disponibile. A Valendas, ad esempio, c'era un gruppo che voleva assolutamente far progredire il villaggio, ma non riusciva ad andare avanti, era bloccato. Molti esterni, tra cui io, hanno collaborato per fare andare le cose nella giusta direzione. Un'iniziativa che nasce dall'interno e un intervento esterno possono creare una combinazione vincente. A Disentis, dove ho lavorato all'abbazia benedettina, ho potuto proporre idee che andassero oltre il singolo edificio. Lo scorso semestre ho sviluppato con i miei studenti alcune idee sull'imminente progettazione urbanistica della comunità di Ilanz - lì il nostro interlocutore era una sindaca attenta e con una formazione da architetto alle spalle. L'obiettivo era elaborare idee diverse per le singole frazioni della grande comunità nata dalla fusione. Le idee nascono dalla percezione, non sono qualcosa di astratto, che si può semplicemente mettere sul tavolo, indipendentemente dal contesto. Ł̀ necessario prima di tutto riconoscere quello che già esiste, per poi essere in grado di percepire ciò che potrebbe essere sviluppato. Inoltre, il coinvolgimento in questi progetti richiede un atteggiamento onesto. All'inizio devo poter dire con convinzione: mi piacciono queste persone, rispetto le loro differenze e i loro punti di vista. Bisogna prima di tutto saper accettare questo. Non puoi venire da fuori e imporre le tue idee alla gente del luogo, come farebbe un missionario. Non piaceresti alle persone e sarebbe una causa persa in partenza.

LS: Dall'introduzione della legge sulle seconde case, all'inizio del 2016, le cose sono cambiate nei Grigioni, e immaginare prospettive di sviluppo basate su un atteggiamento idealistico o filosofico è diventato più difficile. La legge proibisce ai comuni con oltre il $20 \%$ di seconde case - la maggioranza dei Grigioni - di costruire ulteriori abitazioni secondarie. Al fine di mitigare in qualche modo le conseguenze di questa legge in area montana sono state introdotte varie eccezioni, nell'interesse della tutela del paesaggio abitato locale. Ad esempio, gli edifici storici che per la legge precedente non erano destinati ad uso residenziale, ora possono essere riconvertiti in seconde case se considerati "caratteristici del paesaggio abitato" e se la loro conservazione non può essere garantita in nessun altro modo. Gli edifici sono considerati "caratteristici del pae- 


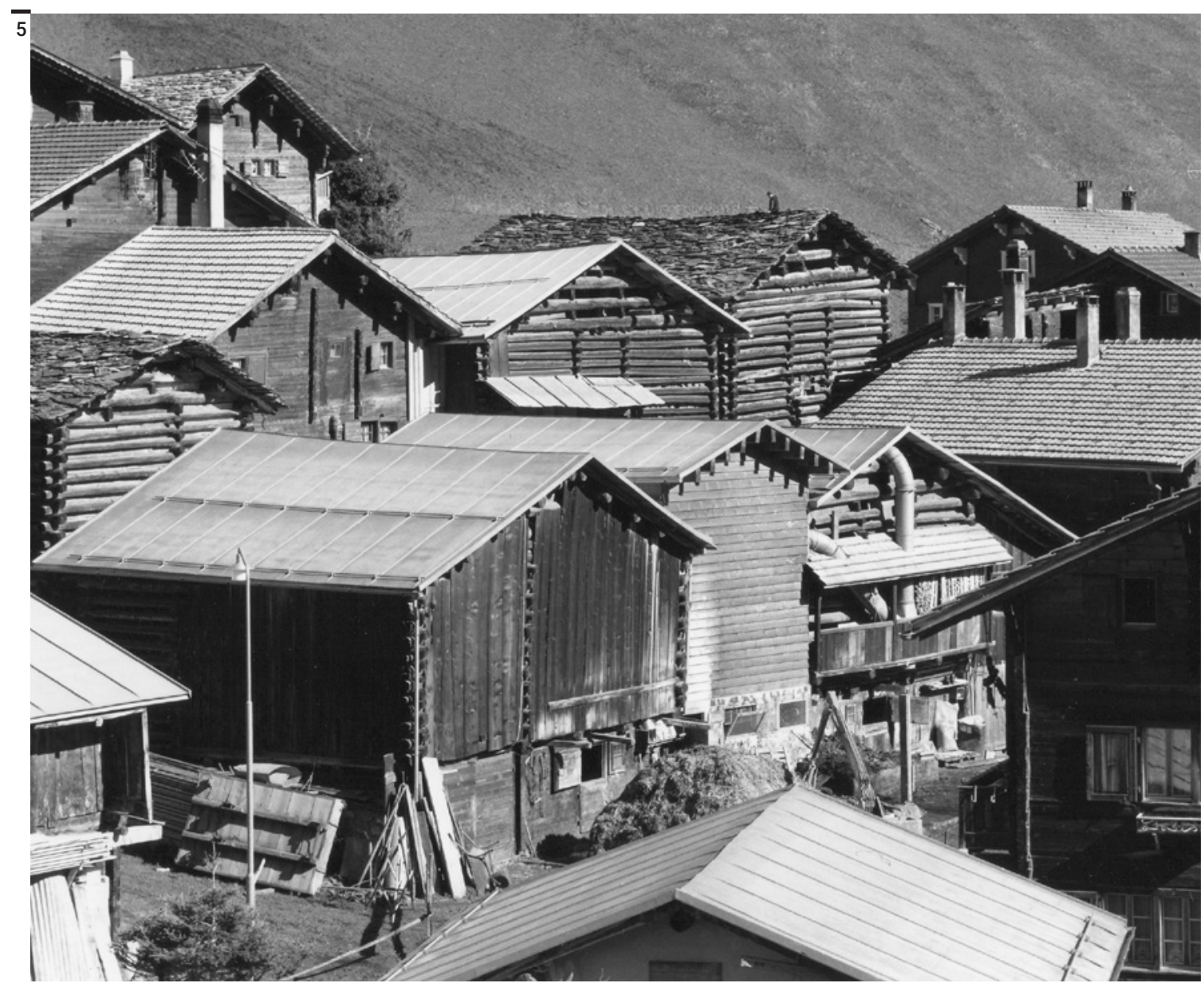

sentlich zur erhaltenswerten Qualität des Ortsbildes und zur Identität des Ortes beitragen.» Dazu gehören bei uns vor allem die Stallscheunen. Sie machen in Graubünden rund die Hälfte des Gebäudebestandes innerhalb der historischen Siedlungskerne aus. Durch den Strukturwandel in der Landwirtschaft sind sie ihrer ursprünglichen Funktion entledigt - und bieten nun bauwilligen Feriengästen eine Möglichkeit, sich ihren Traum vom eigenen Ferienhaus trotz Zweitwohnungsbaustopp doch noch zu erfüllen. Allerdings darf ein ortsbildprägender Bau bei der Umnutzung in seinem Schutzwert nicht beeinträchtigt werden; «insbesondere die äussere Erscheinung und die bauliche Grundstruktur des Gebäudes» müssen, so will es das Gesetz, «im Wesentlichen unverändert bleiben.» Auf einem landwirtschaftlichen Ökonomiebau übertragen heisst dies: Er muss seine historische Funktion nach aussen abbilden, obwohl er nicht mehr zur Lagerung des Winterfutters gebraucht und auch keinem Vieh

Abb. 5 / Fig. 5 Clavau in Vrin, Graubünden / Clavau a Vrin, Grigioni (foto Lucia Degonda). mehr als Unterstand dient, sondern als behagliche Behausung für Menschen einer Wohlstandsgesellschaft eingerichtet ist.

AR: Ja, das ist tatsächlich verheerend. Die meisten Gemeinden tendieren dazu, im Rahmen der Ge- saggio abitato", se "per posizione e forma contribuiscono in modo significativo alla qualità del paesaggio abitato e all'identità del luogo da preservare". Nei nostri territori questo riguarda soprattutto le stalle. Nei Grigioni, le stalle costituiscono circa la metà del patrimonio edilizio esistente all'interno dei centri storici. A causa del cambiamento strutturale nell'agricoltura, le stalle sono state private della loro funzione originale, e oggi offrono ai turisti che vogliono costruire qualcosa l'opportunità di realizzare il sogno di una casa per le vacanze, nonostante il divieto di costruzione di ulteriori abitazioni secondarie. Il restauro di un edificio non può danneggiarne, tuttavia, il valore estetico per il paesaggio abitato locale. La legge specifica che: "In particolare, l'aspetto esteriore e la struttura di base dell'edificio devono rimanere sostanzialmente inalterati". Nel caso di un edificio ad uso agricolo, significa che l'aspetto esteriore deve rifletterne la funzione storica, anche se non è più utilizzato per conservare i foraggi invernali o come riparo per il bestiame e oggi, al contrario, gli interni sono arredati per fungere da confortevole abitazione per i benestanti.

AR: Sì, è davvero un disastro. La maggior parte dei comuni tende ad includere in questa categoria il nu- 


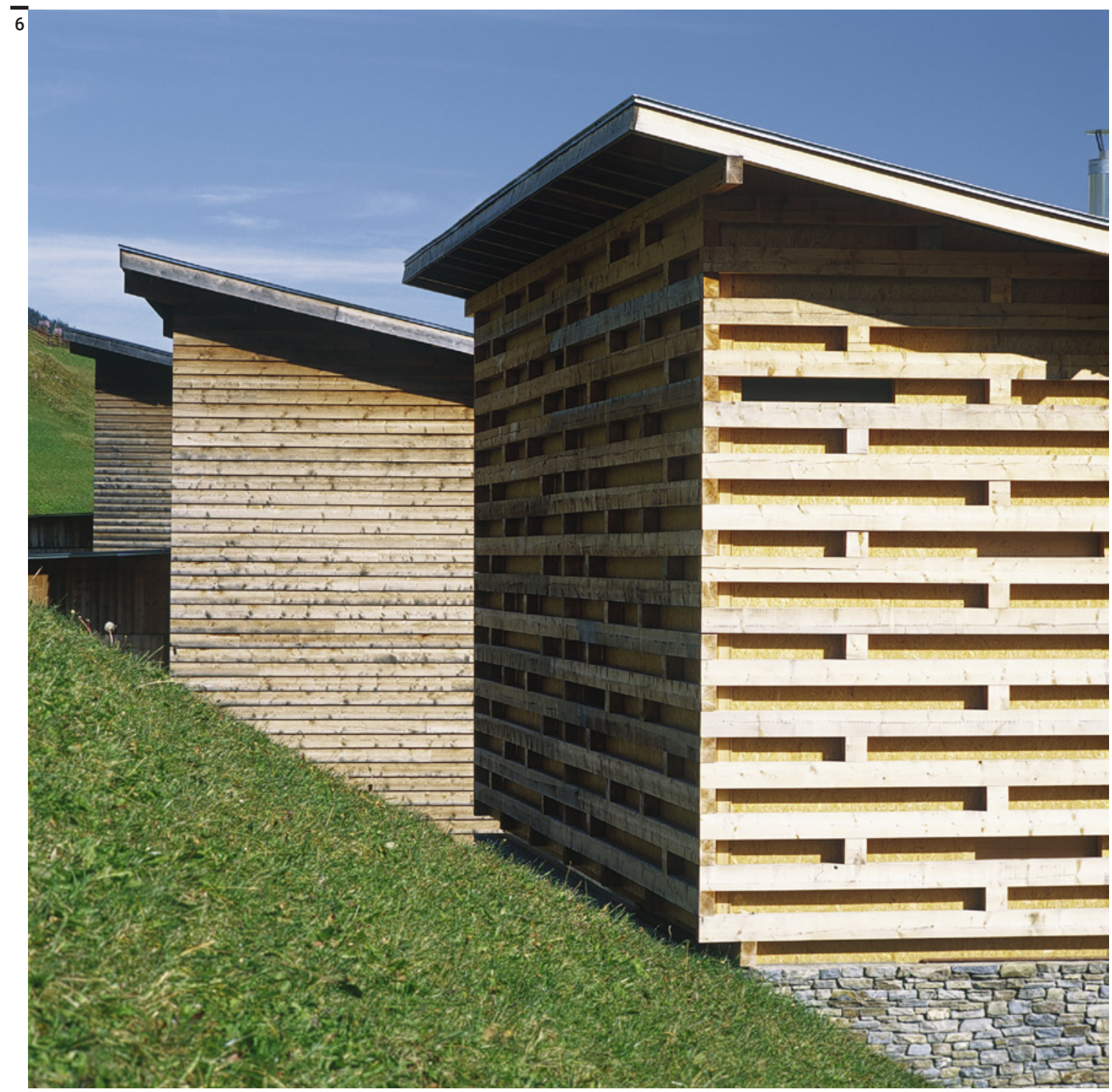

samtschau, die für die Bezeichnung der ortsbildprägenden Bauten in der Grundordnung gefordert wird, möglichst viele der Dorfställe in diese Kategorie aufzunehmen. Bei einem Umbau ist der Architekt dann gezwungen, sich an den Leitfaden der Denkmalpflege zu halten, der eben darauf abzielt, dass das Gebäude auch nach dem Umbau das Bild eines Stalls vermittelt. Da gibt es kaum Spielraum. Das verhindert jede intellektuelle Auseinandersetzung mit der Umnutzungs-Thematik. Kulturell und architektonisch ist das nicht erstrebenswert, da so jede Form von Innovation verhindert wird. Es

Abb. 6 / Fig. 6 Ställe Sut Vitg, Vrin, Graubünden, Gion A. Caminada

/ Stalle Sut Vitg, Vrin, Grigioni, Gion A. Caminada (foto Lucia Degonda) mero più alto possibile di stalle del proprio villaggio, all'interno del quadro complessivo per la designazione degli edifici ritenuti caratteristici del paesaggio abitato locale. In caso di ristrutturazione, l'architetto è quindi costretto a aderire alle linee guida per il restauro di edifici storici, volte appunto a garantire che l'edificio mantenga l'aspetto di una stalla anche dopo la ristrutturazione. Non c'è margine di manovra, e così si impedisce qualsiasi scambio di idee sul tema della riconversione. Dal punto di vista culturale e architettonico, questo atteggiamento non è auspicabile, poiché ostacola qualsiasi forma di innovazione. Preserva un mondo illusorio che in realtà non esiste più.

GG: Anche il comune di Lumnezia sta cercando di classificare il maggior numero possibile di stalle come edifici caratteristici del paesaggio abitato locale. Ovviamente, è un'opportunità allettante per i proprietari, perché edifici apparentemente "privi di
GG: Auch im Lugnez ist man seitens der Gemeinde bestrebt, möglichst viele Dorfställe als ortsbildprägend einzustufen. Für die Stallbesitzer ist das natürlich verlockend, weil damit scheinbar «wertlose» Gebäude zur Kapitalanlage mutieren. In 
Vrin versuche ich Gegensteuer zu geben, indem ich die Leute über die Folgen aufkläre. Diese Ställe sind weit mehr als bloss unternutzte Kulissen für das Ortsbild, die in Zweitwohnungen umgebaut werden können. Man muss sie als ein Privileg der Dörfer erkennen, das die Stadt nicht kennt. Im dichten Dorfkern sind sie kleine Abstandhalter zwischen den privaten Wohnhäusern und eine Art dritter Raum zwischen dem eindeutig öffentlichen und dem rein privaten Raum. Das Dorf setzt sich zusammen aus lauter kleinen Ensembles bestehend aus Haus und Stall und dem Raum dazwischen. Wenn die Ensembles in sich abgeschlossen sind, dann wird's stark, dann können sie zueinander in Beziehung treten. In diesem Sinne sind die Ställe Teil von nachbarschaftlichen Kontexten. Wir können die Ställe als einfache, unkomplizierte Räume ohne festgeschriebenen Zweck betrachten, die je nach Situation, Bedürfnissen und Umständen unterschiedliche Aufgaben und Bedeutungen erhalten können. Das verlieren wir, wenn wir alle Ställe (und Häuser) nur des schnellen Geldes wegen an Auswärtige verkaufen. Ist der Stall einmal «ortsbildprägend» eingestuft, muss auch der Einheimische sich an die rigiden Umbauvorschriften halten. Dann liegt auch ein Umbau in eine Garage oder eine Werkstatt nicht mehr drin. Eine Entwicklung in Richtung von Ensembles mit Entitäten, die für den abgelegenen Ort unabdingbar sind, ist nicht mehr möglich.

AR: Auch der Ersatz des Stalles durch ein Wohnhaus nicht. Letztlich verhindert das eine differenzierte Auseinandersetzung mit der Entwicklung unserer Dörfer. In unseren eng strukturierten, wenig bevölkerten Dörfern beschäftigt mich das Thema des Entdichtens. Vielleicht kann man da und dort einen Stall, statt ihn zu einer Zweitwohnung umzubauen zu einer Dachterrasse umnutzen oder einen Garten oder einen kleinen Platz, um ein bisschen Raum und Luft zu schaffen. Das ist dann nicht mehr möglich.

GC: Ja, auch hier gibt es viel Überzeugungsarbeit zu leisten. Aber es macht uns Spass, weiter dranzubleiben. Und nach uns kommen hoffentlich andere, denen die Zukunft unserer Dörfer nicht gleichgütig ist - auch in architektonischer Hinsicht. valore" diventano un investimento. A Vrin, provo a contrastare questa situazione informando la gente sulle sue conseguenze. Queste stalle sono molto più che semplici scenografie sottoutilizzate per il paesaggio abitato locale, convertibili in seconde case. Devono essere riconosciute come patrimonio dei villaggi montani che le città non conoscono. Nel centro storico del villaggio, più densamente costruito, le stalle sono piccoli distanziatori tra le case private e una sorta di terzo spazio tra quello dichiaratamente pubblico e quello strettamente privato. Il villaggio è costituito da moltissime piccole unità, composte da abitazione, stalla e lo spazio che le divide. Quando queste unità sono autonome, diventano forti e sono in grado di relazionarsi tra loro. In questo senso, le stalle fanno parte anche dei contesti circostanti. Possiamo considerare le stalle come stanze semplici, regolari e senza uno scopo preciso, che possono assumere usi e significati diversi a seconda della situazione, delle esigenze e delle circostanze. Se tutte le stalle (e le abitazioni) vengono vendute a terzi in cambio di denaro facile, finiamo per perdere tutto questo. Una volta che la stalla è stata classificata come "caratteristica del paesaggio abitato", anche il residente del luogo deve rispettare le rigide normative di conservazione secondo cui, ad esempio, la riconversione di una stalla in un garage o un'officina non è più consentita. Di fatto, viene impedito lo sviluppo di unità con funzioni indispensabili in luoghi isolati come questi.

AR: Non sarà più concesso neanche trasformare la stalla in un'abitazione e, in definitiva, questo ostacola un approccio differenziato allo sviluppo dei nostri villaggi. Qui vivono poche persone, ma la struttura dell'abitato è molto densa, e io mi occupo del tema del diradamento urbano. Per fare un esempio, invece di riconvertire una stalla in una seconda casa, potrebbe essere possibile trasformarla in una terrazza, un giardino o un piccolo luogo che crei spazio e aria. Tutto questo non sarà più possibile.

GC: Sì, anche in questo caso sarà necessario un grande sforzo di persuasione. Ma ci appassiona vedere come andrà a finire. $\mathrm{E}$ speriamo che chi verrà dopo di noi non sarà indifferente al futuro dei nostri villaggi - anche dal punto di vista architettonico. 